\title{
COUNTEREXAMPLES CONCERNING BITRIANGULAR OPERATORS
}

\author{
M. S. LAMBROU AND W. E. LONGSTAFF \\ (Communicated by Palle E. T. Jorgensen)
}

\begin{abstract}
An operator on a separable Hilbert space is called bitriangular if it and its adjoint have upper triangular representations with respect to two (perhaps different) orthonormal bases. Although bitriangular operators have some tractable properties and seem to be the right context for generalization of matrices to infinite dimensions, we give counterexamples to various open problems regarding this class of operators. The counterexamples make use of a property that an $M$-basis may or may not have.
\end{abstract}

In their remarkable paper [2] Davidson and Herrero introduce and study the class of bitriangular operators (see below for definitions) on separable Hilbert spaces, giving convincing reasons why they are the best possible extension of direct sums of Jordan blocks (Jordan forms) to infinite dimensional spaces. For example they show that the class of bitriangular operators form the largest class of operators which are quasi-similar to canonical Jordan forms. This class of operators includes the algebraic operators, the diagonal normal operators, the block diagonal operators and others, and members of this class have a rich supply of invariant and hyperinvariant subspaces.

In spite of the plethora of interesting properties of bitriangular operators established in [2], the authors give many examples illustrating the often peculiar nature of such operators. Also in their paper the authors raise various open problems which are known to have affirmative answers in wide classes of special cases. In the present paper we show that for a certain class of bitriangular operators, some of these problems are equivalent to each other and have answers depending upon a property an $M$-basis may or may not have. Thereby we are able to produce counterexamples by choosing the $M$-basis appropriately, and so we add some more peculiarities to this apparently well-behaved class of operators. Larson and Wogen [4] have constructed particular bitriangular operators that answer negatively two of the problems in [2]. We briefly comment on their counterexamples below.

Received by the editors January 8, 1990.

1980 Mathematics Subject Classification (1985 Revision). Primary 47D25, 46B15.

Key words and phrases. Triangular operator, bitriangular operator, invariant subspace, $M$-basis, strong $M$-basis. 
Most of the notation we use here follows that of [2], with which we shall assume some familiarity. In particular $H$ will be a fixed complex separable Hilbert space whose inner product is designated as $\langle\cdot, \cdot\rangle$. Often a wider class of Banach spaces could be considered in place of $H$, but for the sake of simplicity we shall not do so. Recall that a (bounded) operator $T$ on $H$ is called triangular if it has an upper triangular matrix representation with respect to some orthonormal basis on $H$. A more or less complete picture of the spectral structure of a triangular operator is in [3] and it can be seen that an operator $T$ is triangular if and only if $\bigvee\left\{\operatorname{Ker}(T-\lambda)^{k} \mid \lambda \in C, k \geq 1\right\}=H$. The subspace $\bigvee_{\mathrm{Z}^{+}} \operatorname{Ker}(T-\lambda)^{k}$, for an operator $T$ and a scalar $\lambda$, is denoted by $\operatorname{Ker}(T-\lambda)^{\omega}$. Also, for $\Gamma \subseteq \mathbf{C}$ we write $H(T, \Gamma)$ for $\bigvee\left\{\operatorname{Ker}(T-\lambda)^{\omega} \mid \lambda \in \Gamma\right\}$. We shall say that an operator $T$ on $H$ is bitriangular if both $T$ and its adjoint $T^{*}$ are triangular (with respect to two, perhaps different, orthonormal bases). The counterexamples constructed below make use of the concept of an $M$-basis of a Banach space. Recall, for a Banach space $X$ with dual $X^{*}$, a sequence $\left(f_{i}\right)_{1}^{\infty}$ of vectors of $X$ is called an $M$-basis if $V_{\mathbf{Z}^{+}} f_{i}=X$ and there exists a sequence $\left(f_{i}^{*}\right)_{1}^{\infty}$ of elements of $X^{*}$ such that $f_{i}^{*}\left(f_{j}\right)=\delta_{i j}$ with $\left(f_{i}^{*}\right)_{1}^{\infty}$ total in $X$, that is, $\bigcap_{1}^{\infty} \operatorname{Ker} f_{i}^{*}=\{0\}$. An $M$-basis is called strong if it also has the property $\bigvee_{I} f_{i}=\bigcap_{\mathbf{Z}^{+} \backslash I} \operatorname{Ker} f_{i}^{*}$ for all $I \subseteq \mathbf{Z}^{+}$(with Hilbert space notation this becomes $\left.\bigvee_{I} f_{i}=\bigcap_{\mathbf{Z}^{+} \backslash I}\left(f_{i}^{* \perp}\right)\right)$. Not all $M$-bases are strong and examples in Hilbert spaces are known (for example Markus [5, 3.1 ${ }^{0}$ ], and Singer [6, p. 244]). The following equivalences will be used later. The equivalence of (i), (ii), and (iii) (valid for Banach spaces) can be found in [1, Theorem 5.1]. Their equivalence to (iv), when the underlying space is a Hilbert space, is Lemma 3.1 of [5].

Theorem 1. Let $\left(f_{i}\right)_{1}^{\infty}$ be an $M$-basis of a Hilbert space $H$ with biorthogonal sequence $\left(f_{i}^{*}\right)_{1}^{\infty}$. The following are equivalent:

(i) $\left(f_{i}\right)_{1}^{\infty}$ is a strong $M$-basis.

(ii) $\left(\bigvee_{I} f_{i}\right) \cap\left(\bigvee_{J} f_{j}\right)=\bigvee_{I \cap J} f_{i}$ for every pair $I, J$ of subsets of $\mathbf{Z}^{+}$.

(iii) $\bigcap_{\lambda \in \Lambda}\left(\bigvee_{I_{\lambda}} f_{i}\right)=\bigvee_{\bigcap_{\lambda \in \Lambda} I_{\lambda}} f_{i}$ for every family $\left\{I_{\lambda}\right\}_{\Lambda}$ of subsets of $\mathbf{Z}^{+}$.

(iv) $\left(\bigvee_{I} f_{i}\right) \vee\left(\bigvee_{\mathbf{Z}^{+} \backslash I} f_{i}^{*}\right)=H$ for every subset $I$ of $Z^{+}$.

To fix yet another notational symbol, let $\left(f_{i}\right)_{1}^{\infty}$ be an $M$-basis on $H$ and let $\left(\lambda_{i}\right)_{1}^{\infty}$ be a sequence of distinct nonzero scalars converging to zero fast enough so that $\sum_{1}^{\alpha}\left|\lambda_{i}\right|\left\|f_{i}^{*}\right\|\left\|f_{i}\right\|<\infty$. Then $\sum_{1}^{\infty} \lambda_{i} f_{i}^{*} \otimes f_{i}$ converges in norm to a compact operator $T$ (here $p \otimes q$ denotes the operator $x \mapsto\langle x, p\rangle q)$. Sums over subsequences of $\mathbf{Z}^{+}$also define compact operators. Since trivially $T\left(\bigvee_{1}^{n} f_{i}\right) \subseteq \bigvee_{1}^{n} f_{i}$ for every $n \in \mathbf{Z}^{+}$, the orthonormal basis obtained from the $\left(f_{i}\right)$ by the Gram-Schmidt process shows that $T$ is triangular. Since $T^{*}=$ $\sum_{1}^{\infty} \bar{\lambda}_{i} f_{i} \otimes f_{i}^{*}$, a similar argument shows that $T^{*}$ is also triangular; thus $T$ is bitriangular. Such a $T$, for appropriate choices of $\left(f_{i}\right)$, will be the basis 
of our counterexamples. Further note that the point spectrum $\sigma_{p}(T)$ of $T$ is $\left\{\lambda_{i} \mid i \in \mathbf{Z}^{+}\right\}$. The Fredholm-alternative shows that if $\lambda \notin\{0\} \cup\left\{\lambda_{i} \mid i \in \mathbf{Z}^{+}\right\}$, then $T-\lambda$ is invertible. Thus for $\Gamma \subseteq \mathbf{C}$ we have

$$
H(T, \Gamma)=\bigvee_{\lambda_{i} \in \Gamma} f_{i} \text { and } H\left(T^{*}, \Gamma^{*}\right)=\bigvee_{\lambda_{i} \in \Gamma} f_{i}^{*} \quad\left(\text { where } \Gamma^{*}=\{\bar{\lambda} \mid \lambda \in \Gamma\}\right)
$$

We are now in a position to state a theorem that will answer some of the open questions in [2].

Theorem 2. Let $\left(f_{i}\right)_{1}^{\infty}$ be an $M$-basis for $H$ and let $T=\sum_{1}^{\infty} \lambda_{i} f_{i}^{*} \otimes f_{i}$, where $\left(\lambda_{i}\right)_{1}^{\infty}$ is a sequence of nonzero distinct scalars converging to zero and satisfying $\Sigma_{1}^{\alpha}\left|\lambda_{i}\right|\left\|f_{i}^{*}\right\|\left\|f_{i}\right\|<\infty$. The following are equivalent:

(a) For every pair $\Gamma_{1}, \Gamma_{2}$ of subsets of $\mathbf{C}$ we have $H\left(T, \Gamma_{1}\right) \cap H\left(T, \Gamma_{2}\right)=$ $H\left(T, \Gamma_{1} \cap \Gamma_{2}\right)$.

(b) For every family $\left\{\Gamma_{\lambda}\right\}_{\Lambda}$ of subsets of $\mathbf{C}$ we have $\bigcap_{\Lambda} H\left(T, \Gamma_{\lambda}\right)=$ $H\left(T, \bigcap_{\Lambda} \Gamma_{\lambda}\right)$.

(c) For every subset $\Gamma$ of $\mathbf{C}$ we have $H(T, \Gamma) \vee H\left(T^{*}, C \backslash \Gamma^{*}\right)=H$.

(d) For every subset $\Gamma$ of $\mathrm{C}$ we have $H(T, \Gamma)^{\perp}=H\left(T^{*}, C \backslash \Gamma^{*}\right)$.

(e) For every hyperinvariant subspace $M$ of $T$ we have $M=$ $\bigvee_{\lambda \in \sigma_{p}(T)} \bigvee_{n \in \mathbf{Z}^{+}}\left(M \cap \operatorname{Ker}(T-\lambda)^{n}\right)$

(f) For every hyperinvariant subspace $M$ of $T$, the operators $T \mid M$ and $P_{M^{\perp}} T \mid M^{\perp}$ are both bitriangular (where $P_{M^{\perp}}$ denotes the orthogonal projection onto $M^{\perp}$ ).

(g) $\left(f_{i}\right)_{1}^{\infty}$ is a strong $M$-basis.

Proof. The equivalence of (c) and (d) follows from Remark 6.9 of [2], where it is shown using the Euclidean Algorithm that, for all bitriangular operators $T$, we have $H(T, \Gamma)^{\perp} \supseteq H\left(T^{*}, C \backslash \Gamma^{*}\right)$. So equality holds in (d) if and only if the same is true for the inclusion $H(T, \Gamma) \oplus H\left(T^{*}, C \backslash \Gamma^{*}\right) \subseteq H$. A simpler way to see the equivalence of (c) and (d) for our special $T$ (rather than all bitriangular $T$ ) will be given below.

Recall that for our $T$ we have $H(T, \Gamma)=\bigvee_{I} f_{i}$ and $H\left(T^{*}, \Gamma^{*}\right)=\bigvee_{I} f_{i}^{*}$ where $I=\left\{i \in \mathbf{Z}^{+} \mid \lambda_{i} \in \Gamma\right\}$. Thus the equivalence of (a), (b), (c), (d), and (g) simply follows from Theorem 1 and the definition of strong $M$-basis.

Next we show the equivalence of $(\mathrm{e})$ and $(\mathrm{g})$. Suppose that $(\mathrm{g})$ fails. Then by definition there is a subset $I \subseteq \mathbf{Z}^{+}$such that $\bigcap_{\mathbf{Z}^{+} \backslash I}\left(f_{i}^{* \perp}\right) \supset \vee f_{i}$ (where $\supset$ denotes proper inclusion). Set $M=\bigcap_{\mathbf{Z}^{+} \backslash I}\left(f_{i}^{* \perp}\right)$. We claim that $M$ is a hyperinvariant subspace for $T$. Indeed let $A$ be operator commuting with $T$. For every $k \in \mathbf{Z}^{+}$the subspace $\operatorname{Ker}\left(T-\lambda_{k}\right)=\left[f_{k}\right]$ is hyperinvariant for $T$ so there is a scalar $\mu_{k}$ with $A f_{k}=\mu_{k} f_{k}$. We further show that $A^{*} f_{k}^{*}=\bar{\mu}_{k} f_{k}^{*}$. This is so because for every $j$ in $\mathbf{Z}^{+}$we have $\left\langle A^{*} f_{k}^{*}-\bar{\mu}_{k} f_{k}^{*}, f_{j}\right\rangle=\left\langle f_{k}^{*}, A f_{j}-\right.$ $\left.\mu_{k} f_{j}\right\rangle=\left\langle f_{k}^{*},\left(\mu_{j}-\mu_{k}\right) f_{j}\right\rangle$. The latter equals 0 whether or not $j$ equals $k$. Since $\bigvee_{1}^{\infty} f_{i}=H, A^{*} f_{k}^{*}=\bar{\mu}_{k} f_{k}^{*}$ as required. It now follows that $A^{*}$ leaves $M^{\perp}$ 
invariant, so $A$ leaves $M$ invariant. Hence $M$ is hyperinvariant for $T$. Now, if $n \in \mathbf{Z}^{+}$and if $\lambda \in \sigma_{p}(T)$, say $\lambda=\lambda_{j}$, we have $M \cap \operatorname{Ker}(T-\lambda)^{n}=M \cap\left[f_{j}\right]$ which, because of biorthogonality, equals either $\left[f_{j}\right]$ or $\{0\}$ for $j \in I$ or $j \notin I$, respectively. Thus we have the proper inclusion

$$
\bigvee_{\lambda \in \sigma_{p}(T)} \bigvee_{n \geq 1}\left(M \cap \operatorname{Ker}(T-\lambda)^{n}\right)=\bigvee_{I} f_{i} \subset \bigcap_{\mathbf{z}^{+} \backslash I}\left(f_{i}^{* \perp}\right)=M
$$

showing that equality fails in (e).

Conversely, suppose (g) holds. If $M$ is a hyperinvariant subspace for $T$, then as $f_{i}^{*} \otimes f_{i}\left(i \in \mathbf{Z}^{+}\right)$commutes with $T$, it leaves $M$ invariant. Hence $f_{i} \notin M$ implies that $M \subseteq f_{i}^{* \perp}$. Thus if $I=\left\{i \in \mathbf{Z}^{+} \mid f_{i} \in M\right\}$ we must have

$$
\bigvee_{I} f_{i} \subseteq M \subseteq \bigcap_{\mathbf{Z}^{+} \backslash I}\left(f_{i}^{* \perp}\right) \text {. }
$$

But by assumption $\left(f_{i}\right)_{1}^{\infty}$ is a strong $M$-basis, so we have equality above, that is $M=\bigvee_{I} f_{i}$. As before for $n \in \mathbf{Z}^{+}$and $\lambda \in \sigma_{p}(T)$, say $\lambda=\lambda_{j}$, we have $M \cap \operatorname{Ker}(T-\lambda)^{n}=M \cap\left[f_{j}\right]$ which again equals $\left[f_{j}\right]$ or $\{0\}$ for $j \in I$ or not. Therefore

$$
\bigvee_{\lambda \in \sigma_{p}(T)} \bigvee_{n \geq 1}\left(M \cap \operatorname{Ker}(T-\lambda)^{n}\right)=\bigvee_{j \in I} f_{j}=M
$$

It remains to show the equivalence of $(\mathrm{g})$ and $(\mathrm{f})$. If ( $\mathrm{g}$ ) fails, choose $M$ as in the proof of the contrapositive of (e) $\Rightarrow(\mathrm{g})$ above. Then $T \mid M$ fails to be triangular since it can easily be shown that $\bigvee_{\lambda \in C} \operatorname{Ker}(T \mid M-\lambda)^{\omega}=\bigvee_{I} f_{i} \subset M$, showing that (f) fails. (A similar argument, but not required here, shows that all other requirements in (f) also fail). Next we show that $(g)$ implies (f). Let $M$ be hyperinvariant for $T$ and so, as in the proof of $(\mathrm{g}) \Rightarrow(\mathrm{e})$, there is an $I \subseteq \mathbf{Z}^{+}$with $M=\bigvee_{I} f_{i}$. It is easy to verify biorthogonality of $\left(f_{i}\right)_{I}$ and $\left(P_{M} f_{i}^{*}\right)_{I}$. Also clearly $\bigvee_{I} f_{i}=M$ and $\left(P_{M} f_{i}^{*}\right)_{I}$ is total in $M$ (for if $x \in M$ is such that $\left\langle P_{M} f_{i}^{*}, x\right\rangle=0$ for all $i \in I$, then $\left\langle f_{i}^{*}, x\right\rangle=0 \quad(i \in I)$. But also if $j \notin I$, then as $f_{j}^{*} \in M^{\perp}$ we have $\left\langle f_{j}^{*}, x\right\rangle=0$. The totality of $\left(f_{i}^{*}\right)_{\mathbf{Z}^{+}}$shows $x=0$, as required). Finally since $T\left|M=\left(\sum_{I} \lambda_{i} f_{i}^{*} \otimes f_{i}\right)\right| M=\Sigma_{I} \lambda_{i} P_{M} f_{i}^{*} \otimes f_{i}$, and $\left(f_{i}\right)_{I}$ is an $M$-basis for $M$, with biorthogonal family $\left(P_{M} f_{i}^{*}\right)_{I}, T \mid M$ is triangular and so is $(T \mid M)^{*}$. To show that $P_{M^{\perp}} T \mid M^{\perp}$ is also bitriangular we argue as follows. Note $M^{\perp}=\bigvee_{\mathbf{Z}^{+} \backslash I} f_{j}^{*}$. We also show that $\bigvee_{\mathbf{Z}^{+} \backslash I} P_{M^{\perp}} f_{j}=$ $M^{\perp}$. Indeed, if $y \in M^{\perp}$ is such that $\left\langle P_{M^{\perp}} f_{j}, y\right\rangle=0$ for every $j \notin I$ then $\left\langle f_{j}, y\right\rangle=0 \quad(j \notin I)$. But if $i \in I$ we have $f_{i} \in M$, so $\left\langle f_{i}, y\right\rangle=0$ again, showing that $y \in\left(\bigvee_{\mathbf{Z}^{+}} f_{j}\right)^{\perp}=(0)$. Now $\left(P_{M^{\perp}} f_{j}\right)_{\mathbf{Z}^{+} \backslash I}$ and $\left(f_{j}^{*}\right)_{\mathbf{Z}^{+} \backslash I}$ are obviously biorthogonal, so $\left(P_{M^{\perp}} f_{j}\right)_{\mathbf{Z}^{+} \backslash I}$ is an $M$-basis for $M^{\perp}$. Hence $P_{M^{\perp}} T \mid M^{\perp}=\sum_{\mathbf{Z}^{+} \backslash I} \lambda_{j} f_{j}^{*} \otimes\left(P_{M^{\perp}} f_{j}\right)$ is triangular and so is its adjoint, concluding the proof.

Some remarks are now in order: Problems 6.4, 6.10, and 6.11 of [2] ask if statements (f), (c), and (e) respectively are true for all bitriangular $T$. That 
this is not the case follows from Theorem 2 by considering an $M$-basis which is not strong. A special $T$ for negative answers for Problems 6.10 and 6.11 was constructed by Larson and Wogen in [4], but the above has the advantage of showing the equivalence of the two problems for a whole family of $T$ 's. Another question in [2] (see Remark 6.9) asks if for a bitriangular $T$ we have $\bigcap_{\Lambda} H\left(T, \Gamma_{\lambda}\right)=H\left(T, \bigcap_{\Lambda} \Gamma_{\lambda}\right)$ in general, or at least for finite indexing sets $\Lambda$. Again, for the family of $T$ considered above, the two questions are equivalent and have a negative answer for an appropriate choice of $\left(f_{i}\right)_{1}^{\infty}$. Actually a bit more is true. For any bitriangular $T$ (not only of the type considered above), the identity $\bigcap_{\Lambda} H\left(T, \Gamma_{\lambda}\right)=H\left(T, \bigcap_{\Lambda} \Gamma_{\lambda}\right)$ and its finite indexing set $\Lambda$ analogue are equivalent. This follows from [1, Theorem 2.1] with $L_{\gamma}=\operatorname{Ker}(T-\gamma)^{\omega}$.

As a final remark note that for the $T$ considered above, it can easily be shown that if the $M$-basis $\left(f_{i}\right)_{1}^{\infty}$ is strong, then each invariant subspace of $T$ is in fact hyperinvariant. Thus in (e) and ( $\mathrm{f}$ ) of Theorem 2 we may replace, because of their equivalence to $(\mathrm{g})$, "hyperinvariant" by "invariant."

\section{REFERENCES}

1. S. Argyros, M. S. Lambrou, and W. E. Longstaff, Atomic Boolean subspace lattices and applications to the theory of bases, Mem. Amer. Math. Soc. (to appear).

2. K. R. Davidson and D. A. Herrero, The Jordan form of a bitriangular operator (to appear).

3. D. A. Herrero, Approximation of Hilbert space operators, vol. I, Research Notes in Math., vol. 72, Pitman, Boston, London, and Melbourne, 1982.

4. D. R. Larson and W. R. Wogen, Reflexivity properties of $T \oplus 0$, J. Funct. Anal. 92 (1990), 448-467.

5. A. S. Markus, The problem of spectral synthesis for operators with point spectrum, Izv. Math.-USSR 4 (1970), 670-696.

6. I. Singer, Bases in Banach spaces. II, Springer-Verlag, New York, 1981.

Department of Mathematics, University of Crete, Iraklion Crete, Greece

Department of Mathematics, University of Western Australia, Nedlands WA 6009, Australia 